Philosophie ANTIQUE

\section{Philosophie antique}

Problèmes, Renaissances, Usages

19 | 2019

L'épicurisme antique

\title{
Fulvia DE LUISE (éd.), Cittadinanza. Inclusi ed esclusi tra gli antichi e i moderni
}

Trento, Università degli Studi di Trento, 2018 (Studi e Ricerche, 17), 297 p., ISBN 978-88-8443-798-3

\section{Étienne Helmer}

\section{(2) OpenEdition}

1 Journals

Édition électronique

URL : https://journals.openedition.org/philosant/1822

DOI : 10.4000/philosant. 1822

ISSN : 2648-2789

Éditeur

Éditions Vrin

\section{Édition imprimée}

Date de publication : 31 octobre 2019

Pagination : 210-213

ISBN : 978-2-7574-2534-3

ISSN : 1634-4561

\section{Référence électronique}

Étienne Helmer, «Fulvia de LuISE (éd.), Cittadinanza. Inclusi ed esclusi tra gli antichi e i moderni »,

Philosophie antique [En ligne], 19 | 2019, mis en ligne le 20 janvier 2019, consulté le 02 décembre 2022.

URL : http://journals.openedition.org/philosant/1822 ; DOI : https://doi.org/10.4000/philosant.1822

\section{(c) (i) (\$)}

Creative Commons - Attribution - Pas d'Utilisation Commerciale - Pas de Modification 4.0 International - CC BY-NC-ND 4.0

https://creativecommons.org/licenses/by-nc-nd/4.0/ 
principe et refuse la position de l'un au-delà de l'être. Contre cette lecture, il s'agit de montrer que la première hypothèse a un objet réel, ou encore, et comme l'écrit Proclus,

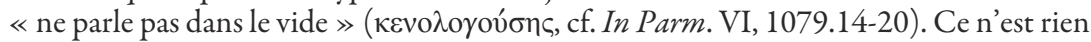
de moins que le geste fondateur du néoplatonisme qui se joue là, soit l'extension de la réalité au-delà de l'être. F. Fauquier en analyse les différentes déclinaisons chez Proclus, encore, mais aussi chez Plotin, Porphyre, Jamblique et Syrianus, tout en montrant comment, une nouvelle fois, cette décision herméneutique implique de faire violence au texte-source et particulièrement de redécouper et réinterpréter la conclusion de la première hypothèse (Parm. 142a6-8) qui paraît l'invalider.

Une troisième et dernière partie, très ample, interroge enfin ce qui constitue le cœur même de l'interprétation néoplatonicienne du Parménide : soit indissociablement la lecture de la première hypothèse comme lieu « où se joue l'exposition d'une transcendance radicale » et la lecture théologique de la deuxième hypothèse. F. Fauquier dégage les trois réquisits d'une telle lecture, ainsi que les différentes modalités de leur mise en œuvre chez Syrianus, Proclus, Jamblique et Damascius : identifier l'un avec le principe de toute chose ; penser que la théologie peut s'exposer sous une forme dialectique et que les négations peuvent soutenir le discours théologique ; enfin, considérer le texte platonicien comme porteur d'une vérité (p. 196). Une vérité d'ailleurs non exclusive puisque - et c'est un point que F. Fauquier interroge également - le néoplatonisme postérieur à Plotin va accorder aux Oracles chaldä̈ques une place elle aussi centrale, s'imposant dès lors de nouvelles « contorsions herméneutiques » de façon à articuler ce texte de structure médioplatonicienne à une hénologie radicale.

Selon la méthode déjà mise en oeuvre, et qui oriente son projet, F. Fauquier s'attache à la fois à analyser en détail la mise en œuvre de ces présupposés herméneutiques, et à souligner la rupture novatrice qu'ils impliquent. De fait, demander si l'on en trouve trace avant Plotin - ainsi, chez Modératus, Numénius, ou les Gnostiques - c'est là encore interroger à nouveaux frais l'originalité du néoplatonisme, la pertinence de sa distinction d'avec le médioplatonisme, et, de ce fait, la pluralité effective des platonismes. À cette question, la réponse de F. Fauquier est claire : si l'exégèse du Parménide est moins centrale chez Plotin que chez ses successeurs, elle est bien radicalement novatrice, et dégage les acquis fondamentaux qui, légués et amplifiés, vont constituer le néoplatonisme en nouvelle « famille spirituelle » et inventer une « configuration métaphysique inédite ». Pour autant, cette lecture théologique est, écrit F. Fauquier, « la moins évidente : elle fait presque violence au texte platonicien dans lequel elle projette une pensée de la transcendance radicale » (p. 428). L'un des grands mérites de cette belle enquête, qui conjugue l'amplitude de champ à la précision de l'analyse (précision dont témoignent encore les études de détail rassemblées en 16 annexes), est d'interroger cette violence qui, parce qu'elle voit en lui la source d'une vérité à extraire et à déployer, fait d'un texte l'origine d'une tradition.

Gwenaëlle Aubry Centre Jean Pépin UMR 8230

Fulvia DE Luise (éd.), Cittadinanza. Inclusi ed esclusi tra gli antichi e i moderni, Trento, Università degli Studi di Trento, 2018 (Studi e Ricerche, 17), 297 p., ISBN 978-88-8443-798-3.

L'homme antique reste souvent identifié à la figure de l'homo politicus, et plus précisément à celle du « citoyen ». Mais que désigne ce terme, transmis jusqu’à nous comme si sa signification n'avait pas changé ? Les neuf textes réunis dans ce volume, 
issus de deux journées d'études qui se sont déroulées à Trente en 2017, montrent, par le biais de comparaisons entre anciens et modernes, comment le vocable unique de « citoyenneté », loin de toute essence éternelle, recouvre des constructions historiques distinctes, dont les enjeux sont parfois explicitement débattus par les « citoyens » eux-mêmes, que ce soit en Grèce ou dans l'Europe moderne. Les contributions, toutes très convaincantes, sont réparties en quatre sections. La première, « Appartenance », porte sur la façon dont la construction d'une identité politique forte a permis de définir l'appartenance civique à la polis classique. Selon F. de Luise, la question de la définition et de l'extension de la citoyenneté dans la démocratie grecque, qui consistait à se demander comment égaliser des inégaux dans un contexte marqué par l'opposition des riches et des pauvres ainsi que par l'héritage aristocratique, a suscité de forts conflits idéologiques. Toutefois, ces conflits ont été dissimulés par l'image unitaire que la démocratie donnait d'elle-même à l'aide d'un certain nombre de mythes - son anti-tyrannie, sa défense de la liberté chez tous les Grecs, celui de l'autochtonie - et d'un geste concomitant d'exclusion de certaines catégories - les femmes, les esclaves, les métèques. C'est la genèse de l'un de ces « mythes d'identité civique » - l'autochtonie - que Silvia Gastaldi restitue, en montrant comment sa signification a évolué, chez les historiens, les poètes et les orateurs, passant de l'occupation originaire du territoire attique à la genèse de ses habitants à partir de la terre elle-même. L'autochtonie a alors servi à définir une sorte d'aristocratie citoyenne fondée sur l'exclusion idéologique des éléments étrangers, à une époque où Athènes revendiquait l'hégémonie sur l'ensemble du monde grec et contre les Perses lors de la seconde guerre médique.

La section suivante, «Identité politique », porte sur la figure du peuple ou demos. Lucilla G. Moliterno explique comment les démagogues en ont construit trois figures principales : le corps civique du peuple souverain, composé à la fois de ceux qui exerçaient une charge et des apragmones qui ne les acceptaient que s'ils y trouvaient un avantage ; les autourgoi, soit ceux qui travaillaient de leurs mains, par opposition à une élite riche, même si l'accent était souvent mis sur la « classe moyenne » plutôt que sur les pauvres; enfin, l'ethnos, c'est-à-dire le peuple envisagé dans son homogénéité culturelle que définit, notamment dans les oraisons funèbres, tout ce qui le distingue des étrangers. Pour l'interprète contemporain, l'enjeu de ces constructions - mais jusqu'à quel point en était-ce ? - qui désignaient le tout ou une partie seulement de la cité, est de comprendre le sens politique d'une pratique de communication politique et ses implications pratiques dans la vie civique. Dans une contribution très documentée comparant les visions antique et moderne du droit, Valentina Pazè soutient, contre l'hypothèse la plus partagée aujourd'hui, que des droits subjectifs existaient en Grèce. Outre des droits politiques, la politeia démocratique assurait en effet aussi aux citoyens des droits assurant leur immunité et leur faculté de s'opposer à ceux qui leur déniaient l'exercice de leurs prérogatives civiques. Bien que les femmes, les esclaves et les métèques en aient été exclus - discrimination fondatrice de la politeia démocratique - on pourrait, selon l'auteure, aller jusqu'à y reconnaître l'idée de droits fondamentaux.

La section suivante est consacrée à l'inclusion et à l'exclusion. Lucio Bertelli suit avec une rare minutie la façon dont Aristote élabore sa définition de la citoyenneté dans les Politiques autour de l'idée de participation. Il en souligne non seulement les variations, entre définition générique et définition spécifique - selon les régimes politiques -, mais aussi les frontières par rapport aux esclaves, aux femmes et aux étrangers, ainsi qu'aux artisans. Ermanno Vitale examine l'origine de la citoyenneté moderne fondée sur le suffrage universel masculin, en retraçant les débats dont cette notion a fait l'objet dans un document anglais du Conseil de la Nouvelle Armée en 1647, puis dans la 
Constitution française de 1793. Le premier de ces deux textes fait état de discussions argumentées qui consistèrent à peser le pour et le contre quant à l'adoption du suffrage universel masculin, et qui portèrent aussi sur le statut de la propriété privée. Le second a suscité des réflexions de personnes comme Olympe de Gouges sur la nécessité d'accorder aussi le droit de vote aux femmes pour que le suffrage universel mérite vraiment son nom. Dans les deux cas, on a prêté plus d'attention à la force éducative de la procédure démocratique et aux transformations sociales qu'elle était en mesure d'apporter, qu'à l'instrumentalisation dont le suffrage universel pouvait aussi faire l'objet - il s'agit davantage d'une préoccupation contemporaine.

Enfin, les trois contributions de la dernière section, intitulée « Les citoyens au-delà de (oltre) la polis », montrent à quel point la citoyenneté antique était liée à des avantages qui n'étaient pas que politiques ou civiques à strictement parler. Deux d'entre elles portent sur la valeur instrumentale que les épicuriens accordent à l'activité politique et, plus généralement, à la sphère politique. Emidio Spinelli se demande si l'on peut dire sans contradiction que les membres du Jardin sont philosophes et citoyens. Contre l'idée encore souvent admise du prétendu rejet de la politique par les épicuriens au nom d'un « vivre caché », son analyse des rapports entre l'utile, le juste, la loi et la philia le conduit à la conclusion qu'il y a une sorte d'intérêt négatif des épicuriens pour la politique : dans la mesure où toutes ces notions visent à assurer les conditions permettant d'éviter le plus possible la douleur, la pratique politique, qui les mobilise, est ainsi perçue comme un moyen d'assurer la sécurité extérieure, et par là, la sérénité intérieure dont dépend le bonheur du sage. Enrico Piergiacomi se penche sur une figure de l'épicurisme romain évoquée dans le De Finibus de Cicéron, Lucius Manlius Torquatus, pour examiner le lien entre vertu et plaisir : si ces deux notions sont intimement liées chez les épicuriens - sous l'égide de la prudence, il n'y a pas de plaisir sans vertu, et la vertu est elle-même plaisante -, cela vaut-il pour ce que la tradition classique, de Solon à Aristote en passant par les sophistes, a défini comme « la vertu civique » ? Comme la contribution précédente, celle-ci conclut à une conception instrumentale du pouvoir pour les épicuriens. Torquatus en particulier interprète la discipline des désirs à la lumière des bénéfices qu'elle apporte pour la stabilité sociale et politique, qu'il conçoit comme des instruments de la félicité dans le cadre d'une analogie entre la cité ou la famille d'un côté, et l'âme humaine de l'autre. L'épicurien est poussé par la sagesse à respecter sa cité et sa famille, car c'est le moyen d'accroître son plaisir, et c'est aussi le cas des vertus comme le courage, la tempérance et la justice. Bien que la vertu civique ne soit pas explicitement mentionnée par Torquatus, il est donc raisonnable de penser que son orientation pratique est identique à celle des autres vertus. Enfin, Silvia Fazzo rouvre le dossier de la citoyenneté romaine d'Alexandre d'Aphrodise, et montre, par l'analyse de la dédicace du philosophe aux empereurs Septime Sévère et Antonin Caracalla dans son traité De Fato, à quel point le privilège de cette citoyenneté octroyée a rendu possible le développement d'une pensée philosophique sans laquelle le poids d'Aristote sur l'Occident médiéval n’aurait sans doute pas été le même.

On peut faire deux critiques à l'ouvrage. La première est que des index des noms, des notions et des passages commentés ou cités auraient été bienvenus pour ajouter à sa valeur d'instrument de recherche. La seconde, plus importante, est qu'il néglige l'un des éléments annoncés dans son titre : s'il traite effectivement des modernes et des anciens, en revanche, les exclus de l'Antiquité y sont moins présents que les inclus. Les femmes, les esclaves, les étrangers et, dans une moindre mesure les artisans, ne sont mentionnés que comme catégories résultant de la limitation de la citoyenneté au mâle athénien ou moderne : aucune contribution n'examine ce que, de leur point de vue, la citoyenneté 
dont ils sont exclus signifie. Néanmoins, ces deux remarques n'entament pas la valeur d'ensemble du livre, dont tout l'intérêt est de mettre à jour des mécanismes et des enjeux significatifs dans la construction d'une notion omniprésente depuis l'Antiquité dans la pensée et la pratique politiques occidentales.

Étienne HeLmer

Université de Porto Rico - etiennehelmer@hotmail.fr

R. Goulet (éd.), Dictionnaire des philosophes antiques, VI, Paris, CNRS éditions, 2016, 1330 p., ISBN 978-2-271-08989-2 ; VII, Paris, CNRS éditions, 2018, 1472 p., ISBN 978-2-271-09024-9.

Avec la publication des tomes VI (2016) et VII (2018) du Dictionnaire desphilosophes antiques, l'entreprise «biobibliographique » colossale menée par Richard Goulet touche à sa fin. On trouve dans ces deux tomes la même rigueur éditoriale et recherche d'exhaustivité que dans les cinq premiers et l'on reste admiratif et reconnaissant devant le travail de coordination, de rédaction et de relecture du maittre d'œuvre. Ce dernier est aussi le principal contributeur en nombre de notices du dictionnaire, étant l'auteur de 135 notices sur les 373 que compte le volumineux tome VI (1330 pages) qui couvre les lettres $S$ et T. Le dictionnaire donne parfois l'impression d'être un incessant « work in progress », comme en témoignent par exemple les « compléments » introduits p. 1273 à 1276 aux notices « Simplicius », « Théano » et «Timarès », ou la double rubrique « iconographie » (p. 180 sq. et 201 sq.) dans la notice consacrée à Sénèque, subtilités formelles qui doivent inciter le lecteur à être très scrupuleux au cours de sa consultation. L'éditeur l'y aide toujours par les nombreux index et renvois internes. Il a également dû composer avec la spécialisation toujours plus grande des champs de recherche : la notice «Socrate » est ainsi répartie entre quatre auteurs différents; sur les cinquante-six pages de la notice, dix-neuf sont consacrées à Socrate lui-même jusqu’à la période hellénistique, trente à la réception de Socrate dans le néoplatonisme et dans la tradition arabe, sept enfin traitent de l'iconographie. La lecture successive de ces quatre parties témoigne de l'importance prise dans nos disciplines par les études de réception et par l'iconographie, mais aussi de conceptions sensiblement différentes du dictionnaire en fonction des rédacteurs, du guide qui oriente avec rigueur, plus proche de la clavis (M. Narcy, p. 415), au guide qui expose (D. Alexandra Layne). Peu importe au demeurant, puisque le guide est bon.

Rien ne laisse présager un tome VIII, puisqu'un Supplément a été publié en 2003 et que le tome VII comprend une rubrique « Compléments » qui intègre de nombreuses notices qui complètent les tomes antérieurs, les lettres $\mathrm{U}$ à $\mathrm{Z}$ n'occupant que le tiers du volume. Parmi les notices, on relèvera, de façon tout à fait subjective, celle consacrée à Varron, qui réévalue l'importance de son œuvre philosophique (pour l'essentiel perdue par la tradition, voir par exemple les pages consacrées à la «théologie tripartite » de Varron), alors qu'on ne retient d'ordinaire que le grammairien ou l'auteur des Res rusticae (p. 94-133), l'importante synthèse de Louis-André Dorion sur Xénophon, définitivement réintroduit dans le cercle des philosophes antiques, celle consacrée à Virgile, apparemment incongrue mais justifiée par l'éducation reçue, les cercles épicuriens fréquentés par le poète, et l'influence sur l'œuvre poétique de l'enseignement mathématique reçu. La notice vertigineuse consacrée à Pythagore, tradition syriaque et arabe incluse, occupe à elle seule 200 pages, auxquelles on doit ajouter 200 autres pages qui traitent de l'histoire de l'école pythagoricienne et de la réception du pythagorisme, 